\title{
Commentary: The lack of a magic bullet
}

\author{
Andrea Montisci, MD, ${ }^{a}$ Silvia Travaglini, $\mathrm{MD},{ }^{\mathrm{b}}$ and Antonio Miceli, $\mathrm{MD}, \mathrm{PhD}^{\mathrm{b}}$ \\ From the Departments of ${ }^{\mathrm{a}}$ Anesthesia and Intensive Care, Cardiothoracic Center and ${ }^{\mathrm{b}}$ Minimally Invasive Cardiac \\ Surgery, Cardiothoracic Center, Istituto Clinico Sant'Ambrogio, Milan, Italy. \\ Disclosures: Authors have nothing to disclose with regard to commercial support. \\ Received for publication Oct 29, 2018; accepted for publication Oct 29, 2018; available ahead of print Dec 12, \\ 2018. \\ Address for reprints: Antonio Miceli, MD, PhD, Istituto Clinico Sant'Ambrogio, Via G.L Faravelli 16, 20149 Mi- \\ lan, Italy (E-mail: antoniomiceli79@alice.it). \\ J Thorac Cardiovasc Surg 2019;157:2352-3 \\ $0022-5223 / \$ 36.00$ \\ Copyright $(\underset{2018}{ } 20 \mathrm{by}$ The American Association for Thoracic Surgery \\ https://doi.org/10.1016/j.jtcvs.2018.10.125
}

Acute kidney injury (AKI) after cardiac surgery is a fearsome complication, affecting up to $30 \%$ of patients undergoing open surgery. ${ }^{1}$ AKI is associated with increased in-hospital mortality, morbidity, and costs. Even minimal changes in creatinine levels are linked with increased risk of worse outcomes. $^{2}$

It is well recognized that its effect on a patient's prognosis is poor in terms of survival and chronic kidney disease development up to 5 years after the initial insult. ${ }^{3}$ This adverse outcome might be due to the late recognition of AKI when the elevation of serum creatinine level is used. In this setting, early detection of impaired renal function in patients may help to ensure more aggressive treatment and to improve clinical outcome.

In this issue of the Journal, Ortega-Loubon and colleagues ${ }^{4}$ propose continuous kidney oxygen saturation monitoring as a noninvasive strategy to improve AKI detection. By using near-infrared spectroscopy, the authors identify the absolute kidney oxygen desaturations below $65 \%$ or declines greater than $20 \%$ from baseline value during the postoperative stage as a new marker for developing postoperative cardiac surgery AKI. Another interesting finding is that declines in kidney regional saturation during surgery were not correlated with postoperative AKI. Although this information is controversial, it adds evidence that AKI is a multifactorial disease and depends on many preoperative, intraoperative, and postoperative variables. To reduce the incidence of postoperative AKI, the authors suggested keeping an adequate perfusion pressure to optimize the cardiac output, enhance hydroelectrolytical replenishment, and avoid nephrotoxic drugs. The novelty of this study is the extension of the monitoring period up to 48 hours after the surgical operation, providing a wider timeframe to identify potential and possibly undetected noxae.

Nevertheless, this study faced the same obstacles of previous reports with near-infrared spectroscopy. The thresholds used to define desaturation are arbitrary, and no dysfunction. $^{7}$

\section{References} 20:187. 2017; 12:e0181158.

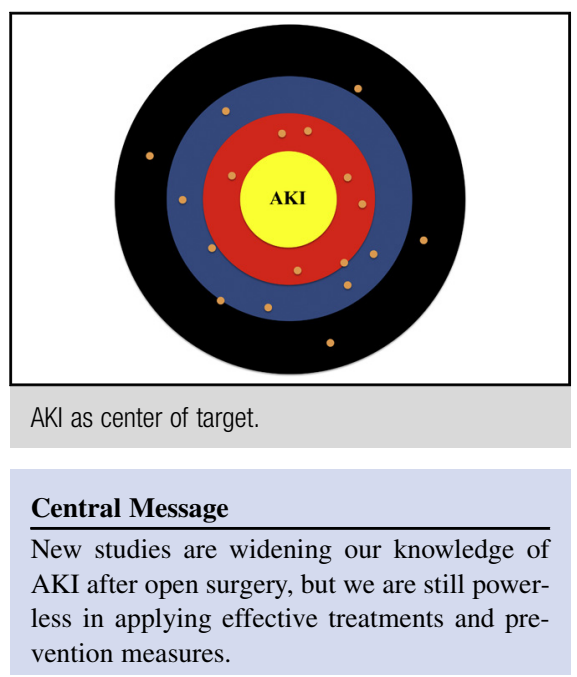

See Article page 2340 .

intervention protocol was used when it was recorded. Furthermore, the authors did not identify the presence of a "window of intervention" to modify outcomes, a major drawback of all studies using regional saturation monitor. ${ }^{5}$ As a result, this system has limited clinical significance. Other monitoring systems during cardiopulmonary bypass have been proposed in detecting AKI. Ranucci and colleagues ${ }^{6}$ showed that the use of goal-directed perfusion protocol, aimed to target intraoperative oxygen delivery to a value greater than $280 \mathrm{~mL} / \mathrm{min} / \mathrm{m}^{2}$, is effective in reducing the less severe stage of AKI. However, it seems to be less effective in reducing the incidence of more severe stages. Promising studies are in progress to identify other potentially treatable triggers, such as right ventricular

The dilemma is that after decades of research on prevention, early detection, and treatment, postoperative AKI is still an orphan of an effective therapy. We still need to find the "magic bullet" to defeat AKI.

1. O'Neal JB, Shaw AD, Billings FT IV. Acute kidney injury following cardiac surgery: current understanding and future directions. Crit Care. 2016;

2. Rosner MH, Okusa MD. Acute kidney injury associated with cardiac surgery. Clin J Am Soc Nephrol. 2006;1:19-32.

3. Ferreiro A, Lombardi R. Acute kidney injury after cardiac surgery is associated with mid-term but not long-term mortality: a cohort-based study. PLoS One. 
4. Ortega-Loubon C, Fernández-Molina M, Fierro I, Jorge-Monjas P, Carrascal Y, Gómez-Herreras JI, et al. Postoperative kidney oxygen saturation as a novel marker for acute kidney injury after adult cardiac surgery. J Thorac Cardiovasc Surg. 2019;157:2340-51.e3.

5. Arrowsmith JE, Ganugapenta MSSR. Intraoperative brain monitoring in cardiac surgery. In: Bonser RS, Pagano D, Haverick A, eds. Brain Protection in Cardiac Surgery. 1st ed. London: Springer-Verlag; 2011:83-111.
6. Ranucci M, Johnson I, Willcox T, Baker RA, Boer C, Baumann A, et al. Goaldirected perfusion to reduce acute kidney injury: a randomized trial. J Thorac Cardiovasc Surg. 2018;156:1918-27.e2.

7. Mertes PM. Venous Congestion and Acute Renal Failure in Cardiac Surgery Postoperative (COVD). Identification No. NCT03013621. Available at: https:// clinicaltrials.gov/ct2/show/NCT03013621?term $=$ right + ventricle $\&$ cond $=$ Acute + Kidney + Injury\&rank=3. Accessed October 20, 2018. 